mean results for sequential scans; rate of decline was unpredictable. The mean change per year was -0.1 z-scores $(S D=$ \pm 0.5 ). Neither Vit D levels nor the CF-related diabetes was related to baseline or changes in BMD.

Conclusion Low lung function and BMI $z$-scores were the greatest indicators of being at risk of osteopaenia for the cohort. With a rate of decline of only $0.1 \mathrm{z}$-score per annum the frequency of DEXA scans could be decreased in low risk children.

\section{P9 A RANDOMISED, DOUBLE-BLIND, PARALLEL GROUP, PLACEBO-CONTROLLED TRIAL OF METFORMIN IN TUBEROUS SCLEROSIS COMPLEX}

${ }^{1} \mathrm{~S}$ Amin, ${ }^{1} \mathrm{AA}$ Mallick, ${ }^{1} \mathrm{H}$ Edwards, ${ }^{1} \mathrm{~A}$ Lux, ${ }^{2} \mathrm{M}$ Laugharne, ${ }^{1} \mathrm{M}$ Likeman, ${ }^{3} \mathrm{~A}$ Khan, ${ }^{4} \mathrm{~F}$ O'Callaghan. ${ }^{\text {P}}$ Paediatric Neurology, University Hospitals Bristol, Bristol, UK; ${ }^{2}$ Radiology, Royal United Hospitals Bath, Bath, UK; ${ }^{3}$ Dermatology, University Hospital North Durham, Durham, UK; ${ }^{4}$ Neurosciences, University College London, London, UK

\subsection{6/archdischild-2018-rcpch.9}

Introduction Tuberous Sclerosis Complex (TSC) is a genetic disorder characterised by the development of benign tumours, secondary to the loss of inhibitory regulation of the mTOR (mammalian Target Of Rapamycin) intracellular growth pathway. Metformin inhibits the mTOR pathway. We investigated the effect of Metformin in patients with TSC.

Methods This was a multicentre randomised, double-blind, parallel group, placebo-controlled trial of metformin in children and adults with TSC. Patients were randomly allocated to placebo or metformin (1:1) for 12 months. The main outcomes were percentage volume change of renal angiomyolipomas (AML) and cerebral Subependymal Giant Cell Astrocytomas (SEGA). Blinded MRI assessments of AMLs and SEGAs were performed at baseline and at 12 months.

Results Fifty-one patients participated, of whom 43 were adults (age range 10-50 years, median age 27, IQR 17). 24 received placebo and 27 received metformin.

The mean AML volume increase from baseline was $25.5 \%$ for the placebo group and $9.6 \%$ for the metformin group. Difference in response, $15.9 \%$ (95\% CI $-9 \%$ to $41 \%$ ) $\mathrm{p}=0.221$.

Twenty-seven patients had SEGAs: 13 received placebo and 14 metformin. The mean SEGA volume increased from baseline by $37 \%$ in the placebo group but reduced from baseline by $23.3 \%$ in the metformin group. Difference in response, $60.3 \%$ (95\% CI $-0.4 \%$ to $111, \mathrm{p}=0.048)$.

Three serious adverse events occurred that reflected the underlying disease. Two were AML haemorrhages and one was worsening seizures requiring hospitalisation.

Conclusions Metformin is safe and well tolerated in children and adults with TSC. Patients on metformin had a significant reduction in SEGA volume compared with placebo. Metformin did not reduce AML size but growth appeared slower than in the placebo group, although this difference was not statistically significant. There may be a role for metformin in slowing or reversing the growth of life-threatening hamartomas in TSC. Further study is justified.

\section{Young People's Health Special Interest Group}

\section{G10 ARE WE USING THE HEEADSSS ASSESSMENT TO SCREEN FOR PSYCHOSOCIAL RISKS WHEN YOUNG PEOPLE ARE ADMITTED TO HOSITAL?}

AE Richardson, M Cooper, DM Wood. Department of Paediatrics, Nottingham Children's Hospital, Nottingham University Hospitals, Nottingham, UK

\subsection{6/archdischild-2018-rcpch. 10}

Aims To evaluate the use of an adolescent psychosocial screening tool (HEEADSSS assessment) on admission to hospital. To identify barriers to screening and inform service development. Methods A retrospective evaluation of a systematic sample of 10-18 year olds admitted over a six month period. Case notes were reviewed to identify the recording of the components of the HEEADSSS assessment. We included an equivalent number of young people admitted with mental health as well as physical health presentations. We asked the medical team to identify barriers to using the HEEADSSS tool when admitting young people to the acute paediatric ward via an electronic survey.

Results We identified 60 young people (mean 13.9 years), 30 admitted with a mental health presentation, and 30 with 'other' acute presentations. Overall 25\% (15/60) had a fully completed HEEADSSS tool, with 57\% (34/60) partially completed, and absent in $18 \%(11 / 60)$. In young people presenting with mental health problems $47 \%$ (14/30) had a completed HEEADSSS, occurring in only $3 \%$ ( $1 / 30$ patient) in the 'other' category (chi squared $15.02 \mathrm{p}<0.0001$ ). In the 'other' group $18 / 30(60 \%)$ had a partially completed HEEADSSS, and absent in $37 \%$. Of those presenting with a 'mental health' problem, $16 / 30$ (53\%) had a partially completed assessment (Fisher's Exact Test $p=0.0001$ ). Overall the components least frequently documented were Eating (13\%), Safety (30\%), Activities (35\%) and Sex (37\%). Barriers to completion included family members being present, the engagement of the young person, lack of familiarity/training using HEEADSSS, and the hospital environment.

Conclusion Our results showed adolescent psychosocial screening is only completed in 1 in 4 young people. The proportion of completed screening was higher in young people presenting with mental health problems compared to those with other presentations. These differences remain when partially completed screens are included. Some parts of the assessment were more frequently completed than others. Barriers to use by admitting clinicians included factors relating both to clinician knowledge and skills, and the hospital environment. These results will assist in clinician training as well as quality improvement for young people admitted to hospital. 